\title{
Contemporary approaches to soft tissue and bone pathology
}

\author{
Khin Thway ${ }^{1}$ - Andrew L. Folpe ${ }^{2}$
}

Published online: 18 December 2019

(C) Springer-Verlag GmbH Germany, part of Springer Nature 2019

Mesenchymal neoplasms are diagnostically challenging, may occur at any anatomic site, and frequently show morphologic and immunophenotypical overlap with other mesenchymal tumors, and with non-mesenchymal lesions. The increasing sophistication of molecular genetic techniques has led to rapid developments in our understanding of the underlying biology of soft tissue and bone tumors, with continued refinements in characterization and biologic stratification, including prediction for entry into specific tailored treatment protocols. This Annual Review issue aims to highlight the current, comprehensive state of the art for various mesenchymal tumor types. Each article in this special issue discusses the biology and line of differentiation taken by these neoplasms, the most salient histomorphologic issues, practical applications of the most upto-date immunohistochemical and molecular developments, and the increasing role of the soft tissue and/or bone pathologist in directing targeted therapies.

Our opening article (https://doi.org/10.1007/s00428-01902678-7) summarizes important recent advances in the immunohistochemical and molecular genetic diagnosis of selected soft tissue tumors, emphasizing their applications in routine diagnosis, the ways in which they have helped to refine tumor classification and treatment stratification, as well as their limitations and pitfalls.

Papke and Hornick (https://doi.org/10.1007/s00428-01902651-4) discuss the current status of vascular neoplasms, a diagnostically challenging tumor group that includes morphologically non-vasoformative variants, may show significant histologic overlap, and frequently poses difficulty in

Khin Thway

khin.thway@rmh.nhs.uk

Andrew L. Folpe

folpe.andrew@mayo.edu

1 Sarcoma Unit, The Royal Marsden Hospital, London SW3 6JJ, UK

2 Department of Laboratory Medicine and Pathology, Mayo Clinic, Rochester, MN 55905, USA accurate assignment to diagnostic and behavioral categories (benign; intermediate, locally aggressive; intermediate, rarely metastasizing; and malignant). Morphologic pointers to diagnosis are summarized, as well as important advances in ancillary testing for this group of neoplasms.

Adipocytic tumors are frequently encountered in routine surgical pathology practice, with atypical and malignant adipocytic neoplasms representing approximately $20 \%$ of all sarcomas. The morphologic differences between lipoma variants and well-differentiated liposarcomas can be subtle, and moreover, there is a heterogeneous group of low-grade spindle cell tumors of differentiated adipose tissue that have been difficult to classify. Creytens discusses the salient histologic and molecular features of the newly proposed group of "atypical spindle cell/pleomorphic lipomatous tumor" (https://doi. org/10.1007/s00428-019-02652-3), as well as those of the recently described and very rare pleomorphic myxoid liposarcoma (myxoid pleomorphic liposarcoma), an apparently novel subtype of liposarcoma with aggressive behavior, which typically occurs in children and young adults with a mediastinal predilection.

Fibroblastic neoplasms encompass a complex spectrum of morphologically overlapping and behaviorally diverse entities, but this group has benefited considerably from the insights obtained from the molecular field, which has allowed significant refinement in tumor characterization and potential treatment stratification. The review by Armstrong and Demicco (https://doi.org/10.1007/s00428-019-02682-x) elegantly details the features of the principal new entities in this group, including EWSR1-SMAD3-rearranged fibroblastic tumors, superficial CD34-positive fibroblastic tumor, and the ever-expanding group of kinase-rearranged sarcomas, including NTRK (neurotropic receptor tyrosine kinase)-rearranged neoplasms.

The complex, overlapping territories of neoplasms with myoid, myofibroblastic, and pericytic differentiation have always been challenging in the setting of routine morphology and immunohistochemistry, given our limited understanding of their molecular background. John's and Fritchie's review 
on these subgroups (https://doi.org/10.1007/s00428-01902700-y) offers a useful breakdown of each subtype, including cellular function and the current status of molecular genetics, and discusses PDGFRB alterations and the spectrum of myopericytoma/myopericytomatosis, $S R F$ RELA fusions in a subset of cellular myofibromas/ myopericytomas, and novel genetic findings in glomus tumors. These insights clearly highlight the different pathogenetic mechanisms for these morphologically overlapping neoplasms.

In their review on new developments in nerve sheath neoplasms, Meyer and Billings (https://doi.org/10.1007/s00428019-02671-0) neatly break down the chief diagnostic and molecular characteristics of principal entities in this group. In the setting of patients with NF1, the accurate diagnosis of neurofibroma, atypical neurofibroma, and low-grade malignant peripheral nerve sheath tumor has always been highly challenging and is often inconsistent between pathologists. These categories are thought to reflect a genetic spectrum, and this review includes discussion of the newly proposed term "atypical neurofibromatous neoplasm of uncertain biologic potential (ANNUBP)," reached by expert consensus and offering criteria for the means of most accurate diagnosis of the spectrum of neurofibromatous lesions seen in NF1 in a multidisciplinary team setting.

An ever-present challenge in the diagnosis of mesenchymal tumors is in their distinction from more common entities such as carcinoma. Agaimy discusses the current biologic concepts of various epithelioid soft tissue neoplasms (https://doi.org/ 10.1007/s00428-019-02677-8), starting with the expanding field of SWI/SNF-deficient tumors, with emphasis on new entities and treatment strategies, then focusing on epithelioid tumors driven by rarer, characteristic genetic abnormalities such as sclerosing epithelioid fibrosarcoma and the recently described GLI1-related malignant epithelioid soft tissue neoplasms.

The molecular spectrum of rhabdomyosarcoma is more varied than previously anticipated, and while these are all typically aggressive neoplasms, there is variation in behavior between specific subclasses. Leiner and Le Loarer (https://doi. org/10.1007/s00428-019-02676-9) offer an update on recently characterized molecular subtypes of rhabdomyosarcoma, such as those harboring MYOD1 mutations, and those with TFCP2- or VGLL2-NCOA2 fusions. Similarly, Sbaraglia, Righi, Gambarotti, and Dei Tos (https://doi.org/10.1007/s00428-019-02720-8) give a contemporary review of the large, markedly heterogeneous group of Ewing and Ewing-like neoplasms, incorporating the recently defined $C I C$ - and BCOR-associated Ewing sarcoma-like neoplasms and emerging classes of neoplasms associated with EWSRI and non-ETS family genes such as NFATC2- and PATZ1-rearranged sarcomas, with salient distinguishing morphologic and clinical characteristics.

Kao, Lee, and Huang (https://doi.org/10.1007/s00428-01902679-6) focus on the complex, heterogeneous spectrum of various chondroid or osteoid/osseous matrix-producing neoplasms, including clinical correlations and the associated molecular background, while Venneker, Szuhai, Hogendoorn, and Bovée (https://doi.org/10.1007/s00428-019-02699-2) shed light on the epigenetics of bone and cartilaginous neoplasms, secondary to recent findings of specific driver mutations identified in chondroblastoma and giant cell tumor of the bone and central cartilaginous tumors (enchondroma and central chondrosarcoma), with reference to the diagnostic impact of these genome-wide discoveries. Franceschini, Lam, Cleton-Jansen, and Bovée (https://doi. org/10.1007/s00428-019-02683-w) give a detailed, clinical, histologic, and molecular synopsis on two examples of bone-forming neoplasms of the skeleton with contrasting genetic backgrounds; osteoid osteomas/osteoblastomas, which have simple karytopes and are translocation-driven, and high-grade osteosarcomas, which have complex karyotypes. Cleven, Shreuder, Groen, Kroon, and Baumhoer (https://doi. org/10.1007/s00428-019-02726-2) focus specifically on mesenchymal neoplasms of the maxillofacial bones, detailing the clinicopathologic and molecular parameters that aid diagnosis and potential avenues for precision medicine.

We very much hope that these articles will provide surgical pathologists with a comprehensive update on the most recent advances in the pathologic and molecular landscape of various mesenchymal neoplasms, and will also be of use to clinicians and scientists who help to take care of sarcoma patients. Soft tissue/bone pathology is an ever-changing and exciting discipline, and it has been our privilege to co-edit this special issue of Virchow's Archiv.

\section{Compliance with ethical standards}

Conflict of interest The authors declare that they have no conflict of interest.

Publisher's note Springer Nature remains neutral with regard to jurisdictional claims in published maps and institutional affiliations. 